\title{
Clinical effectiveness of mupirocin for preventing $S$. aureus infections in non-surgical settings: a meta-analysis
}

\author{
R Nair ${ }^{1}$, E Perencevich ${ }^{2}$, A Blevins ${ }^{3}, M$ Goto ${ }^{2}$, R Nelson ${ }^{4}$, ML Schweizer ${ }^{2 *}$ \\ From 3rd International Conference on Prevention and Infection Control (ICPIC 2015) \\ Geneva, Switzerland. 16-19 June 2015
}

\section{Introduction}

A protective effect of mupirocin has been seen among surgical, nonsurgical and dialysis patients. Our aim is to summarize evidence for mupirocin decolonization for prevention of $S$. aureus infections in non-surgical healthcare settings.

\section{Objectives}

To identify the optimal setting and patient population to implement mupirocin decolonization for prevention of $S$. aureus infections using meta-analytic methods.

\section{Methods}

We conducted systematic searches in PubMed, Cochrane Library Databases, Scopus, Web of Science, and ClinicalTrials.gov to identify papers published until 2013 on effectiveness of mupirocin in healthcare settings. Two investigators independently abstracted data with a pilottested form. Risk of bias was assessed using the Cochrane tool. The crude odds ratios were pooled (cpOR) using a random-effects model. Heterogeneity was evaluated using the Woolf's test for homogeneity and $I^{2}$ statistics.

\section{Results}

Of the 12,644 studies identified, 8 randomized controlled trials and 19 quasi-experimental studies met the study inclusion criteria. Mupirocin was observed to reduce the odds of $S$. aureus infections by $70 \%(\mathrm{cpOR}=0.30,95 \% \mathrm{CI}$ $0.23,0.39)$ and $60 \%(\mathrm{cpOR}=0.40,95 \%$ CI $0.27,0.62)$ in both dialysis and non-dialysis settings, respectively. Nevertheless, there was highly significant $(\mathrm{p}=0.0009)$ and moderate heterogeneity $\left(I^{2}=46 \%\right)$ among studies. Studies were homogeneous ( $p>0.1)$ when stratified analyses were performed by specific clinical settings. Among the $6 \mathrm{stu}-$ dies that took place in adult intensive care units (ICUs), mupirocin decolonization was associated with a $56 \%$ reduction in the odds of $S$. aureus infection (cpOR $=0.44$, $95 \%$ CI $0.26,0.73$ ). There was also a protective effect of mupirocin against $S$. aureus exit site infections among patients undergoing peritoneal dialysis $(\mathrm{cpOR}=0.23,95 \%$ CI $0.15,0.36$ ) and against bacteremia among hemodialysis patients (cPOR $=0.15,95 \%$ CI $0.06,0.36)$.

\section{Conclusion}

Mupirocin decolonization is protective against $S$. aureus infections among both dialysis and adult ICU patient populations. Future studies should target other patient settings such as long-term care facilities.

\section{Disclosure of interest}

None declared.

\section{Authors' details \\ ${ }^{1}$ Epidemiology, University of lowa, lowa City, IA, USA. ${ }^{2}$ Internal Medicine, University of lowa, lowa City, IA, USA. ${ }^{3}$ Library Sciences, University of lowa, Iowa City, IA, USA. ${ }^{4}$ Internal Medicine, University of Utah, Salt Lake City, UT, USA.}

Published: 16 June 2015

doi:10.1186/2047-2994-4-S1-O5

Cite this article as: Nair et al:: Clinical effectiveness of mupirocin for preventing $\mathrm{S}$. aureus infections in non-surgical settings: a meta-analysis. Antimicrobial Resistance and Infection Control 2015 4(Suppl 1):O5.

${ }^{2}$ Internal Medicine, University of lowa, lowa City, IA, USA

Full list of author information is available at the end of the article 